\title{
Does additional coronary artery bypass grafting to aortic valve replacement in elderly patients affect the early and long-term outcome?
}

\author{
Francesco Formica ${ }^{1}$. Serena Mariani ${ }^{1}$. Stefano D'Alessandro ${ }^{1}$. Gurmeet Singh ${ }^{2}$. Michele Di Mauro ${ }^{3}$. \\ Maria Grazia Cerrito ${ }^{4}$ Luigi Amerigo Messina ${ }^{4,5} \cdot$ Salvatore Scianna $^{1} \cdot$ Francesca Papesso $^{1} \cdot$ Fabio Sangalli $^{6}$
}

Received: 8 March 2019 / Accepted: 2 October 2019 / Published online: 18 October 2019

(c) Springer Japan KK, part of Springer Nature 2019

\begin{abstract}
Early and long-term outcomes in elderly patients who underwent isolated aortic valve replacement (iAVR) are well defined. Conflicting data exist in elderly patients who underwent AVR plus coronary artery bypass grafting (CABG). We sought to evaluate the early and long-term outcomes of combined AVR + CABG in patients older than 75 years of age. From June 1999 to June 2018, 402 patients $\geq 75$ years who underwent iAVR $(n=200 ; 49.7 \%)$ or combined AVR plus CABG $(n=202$; $50.3 \%$ ) were retrospectively analysed. AVR + CABG patients were older than iAVR patients ( $78.5 \pm 2.5$ vs $77.6 \pm 2.8$ years; $p<0.0001$ ), with greater co-morbidities and more urgent/emergency surgery. 30-day mortality was $6.5 \%$ in the AVR+CABG and $4.5 \%$ in the iAVR group $(p=0.38)$. Multivariate analysis identified EuroSCORE II [odd ratio (OR) 1.13] postoperative stroke (OR 12.53), postoperative low cardiac output syndrome (OR 8.72) and postoperative mechanical ventilation $>48 \mathrm{~h}$ (OR 8.92) as independent predictors of 30-day mortality; preoperative cerebrovascular events (OR 3.43), creatinine (OR 7.27) and extracorporeal circulation time (OR 1.01) were independent predictors of in-hospital major adverse cardiovascular and cerebral events (MACCE). Treatment was not an independent predictor of 30-day mortality and in-hospital MACCE. Survival at 1,5 and 10 years was $94.7 \pm 1.6 \%, 72.6 \pm 3.6 \%$ and $31.7 \pm 4.8 \%$ for iAVR patients and $89.1 \pm 2.3 \%, 73.9 \pm 3.5 \%$ and $37.2 \pm 4.8 \%$ for AVR + CABG subjects $(p=0.99)$. Using adjusted Cox regression model, creatinine [hazard ration (HR) $1.50 ; p=0.018$ ], COPD (HR 1.97; $p=0.003$ ) and NYHA class (HR 1.39; $p<0.0001$ ) were independent predictors of late mortality; the combined AVR + CABG was not associated with increased risk of late mortality (HR $0.83 ; p=0.30)$. In patients aged $\geq 75$ years, combined AVR + CABG was not associated with increased 30-day mortality, in-hospital MACCE and long-term mortality. Surgical revascularization can be safely undertaken at the time of AVR in elderly patients.
\end{abstract}

Keywords Elderly $\cdot$ AVR $\cdot$ CABG $\cdot$ Outcomes

Francesco Formica

francesco_formica@fastwebnet.it

1 Department of Medicine and Surgery, Cardiac Surgery Clinic, San Gerardo Hospital, University of Milano-Bicocca, Via G.B. Pergolesi 33, 20052 Monza, Italy

2 Department of Critical Care Medicine and Division of Cardiac Surgery, Department of Surgery, Mazankowski Alberta Heart Institute, University of Alberta, Edmonton, Canada

3 Heart Department, SS Annunziata Hospital, Chieti, Italy

4 Department of Medicine and Surgery, University of Milano-Bicocca, Monza, Italy

5 Statistics and Quality Health Service, ASST San Gerardo Hospital, Monza, Italy

6 Department of Anaesthesia and Intensive Care Medicine, San Gerardo Hospital, Monza, Italy

\section{Introduction}

The World Health Organization determined that patients aged 80 years or more will number approximately 400 million by 2050 [1]. In Europe, the elderly population ( $>65$ years old) is estimated to approach $28.5 \%$ in 2050 compared to $19.2 \%$ in 2016 [2]. The aging population correlates with an increasing prevalence of aortic valve disease, primarily aortic stenosis (AS). Thus, it is anticipated that in the future greater numbers of elderly patients will require aortic valve replacement (AVR). From 1999 to 2011, the rate of AVR per 100,000 person-year increased, with a significant increment in patients $>75$ years old [3].

Among elderly patients referred for AVR, the rate of coronary artery disease (CAD) ranges between 40 and $70 \%$ 
[3-5], and approximately one-half of patients require coronary artery bypass grafting (CABG) in addition to AVR [6]. Medical therapy cannot improve patients with symptomatic aortic valve disease, and surgical AVR remains the gold standard for patients with low and intermediate operative risk. It is strongly recommended that concomitant CABG is performed at the time of AVR for critical (> 70\%) coronary stenosis [6].

Transcatheter aortic valve implantation (TAVI) provides an option for elderly and high-risk patients who otherwise may be excluded from conventional surgical AVR. In intermediate-risk patients, TAVI has lower 30-day mortality and stroke reported compared to conventional AVR [7]. Although TAVI results to date are encouraging, there remain significant limitations. The procedure is technically demanding, especially in the setting of aggressive peripheral arteriopathy. Furthermore, significant noteworthy complications, such as perivalvular leak, requirement for permanent pacemaker implantation, acute kidney injury, and embolic stroke remain concerns [8].

While early and late results in elderly patients undergoing isolated AVR are homogeneous, the early and late outcomes of combined AVR with CABG are still unclear and sometimes conflicting. In elderly patients, AVR with CABG has been reported to be associated with early mortality ranging from 1.9 to 24\% [9-12] compared to AVR alone (ranging from 0 to $7.6 \%$ ) [13-17]. In the overall population, simultaneous AVR and CABG have worse long-term survival compared to isolated AVR [18], while among the elderly population, survival between the two treatments appears to be comparable [19-21].

This study sought to investigate whether combined AVR and $\mathrm{CABG}$ increases early mortality and morbidity and long-term survival compared with isolated AVR in elderly patients aged more than 75 years.

\section{Materials and methods}

Retrospective evaluation of prospectively collected data from the local surgery database at the San Gerardo Hospital, University of Milano-Bicocca (Monza, Italy), was conducted. From June 1, 1999, to Jun 31, 2018, 1284 patients underwent isolated AVR (iAVR) or AVR combined with other cardiac surgical procedures. 402 elderly ( $\geq 75$ years) patients underwent iAVR or combined with CABG. Figure 1 shows both the total number of patients and the patients of the study population operated year by year during the study period. The subjects were divided into two groups according to the type of the operation: iAVR group $(n=200 ; 49.7 \%)$ and AVR + CABG group $(n=202 ; 50.3 \%)$. Patients who underwent emergency or salvage operation were excluded from the analysis.
All patients were examined with two-dimensional and Doppler echocardiography and coronary angiogram. The local ethical committee approved the Myocardial REvascularization associated with Aortic valve replacement in the Long-TERM survival in the elderly population (My-REALTERM) study. Individual patient consent was waived (EC resolution n. 1560, 17th October 2017). Primary study end points were early and late all-cause mortality.

\section{Variable definitions}

All variables included in the EuroSCORE II model were included. Atrial fibrillation was divided into paroxysmal or persistent/permanent. Urgent operations were procedures performed in patients whose symptoms dramatically worsened, or stable patients with severe coronary anatomy, or haemodynamically unstable patients requiring inotropes. Urgent operations were performed during the same hospital stay as the referral.

All patients underwent preoperative coronary angiogram and echocardiography. Left main disease was defined as stenosis greater than $50 \%$. Critical coronary disease was defined as stenosis greater than $70 \%$, or more the $60 \%$ in the proximal left anterior descending artery.

Regarding perioperative variables, stroke was defined as an episode of neurological dysfunction caused by focal cerebral, spinal, or retinal infarction that occurred in the perioperative or postoperative period with symptoms remaining evident for more than $24 \mathrm{~h}$ and with imaging evidence of brain infarction. Transient ischemic attack (TIA) was defined as a transient neurological event with loss of neurological function during less than $24 \mathrm{~h}$. Perioperative myocardial infarction was defined if two of the three following signs were simultaneously recorded within $72 \mathrm{~h}$ after the operations: new Q-waves on electrocardiography, new hypokinesia or akinesia myocardial segments at echocardiogram or abnormal release of creatine kinase MB more than five times the upper limit of normal value (25 IU/l). Low cardiac output syndrome was defined as the need for inotropes and or intra-aortic balloon pump. Respiratory failure was defined as the need for prolonged mechanical ventilation more than $48 \mathrm{~h}$ or the requirement of tracheostomy. Major adverse cardiovascular and cerebral events (MACCE) were defined as operative mortality, stroke, new myocardial infarction and acute renal failure. Re-intervention was defined as an operation during the same hospital stay because of postoperative bleeding or cardiac tamponade. Operative mortality was defined as any death occurring within 30 days from the time of surgery if the patients had been discharged from the hospital, or any death occurring during the initial hospital stay regardless of the time from the operation. 


\section{Number of Operations}

100
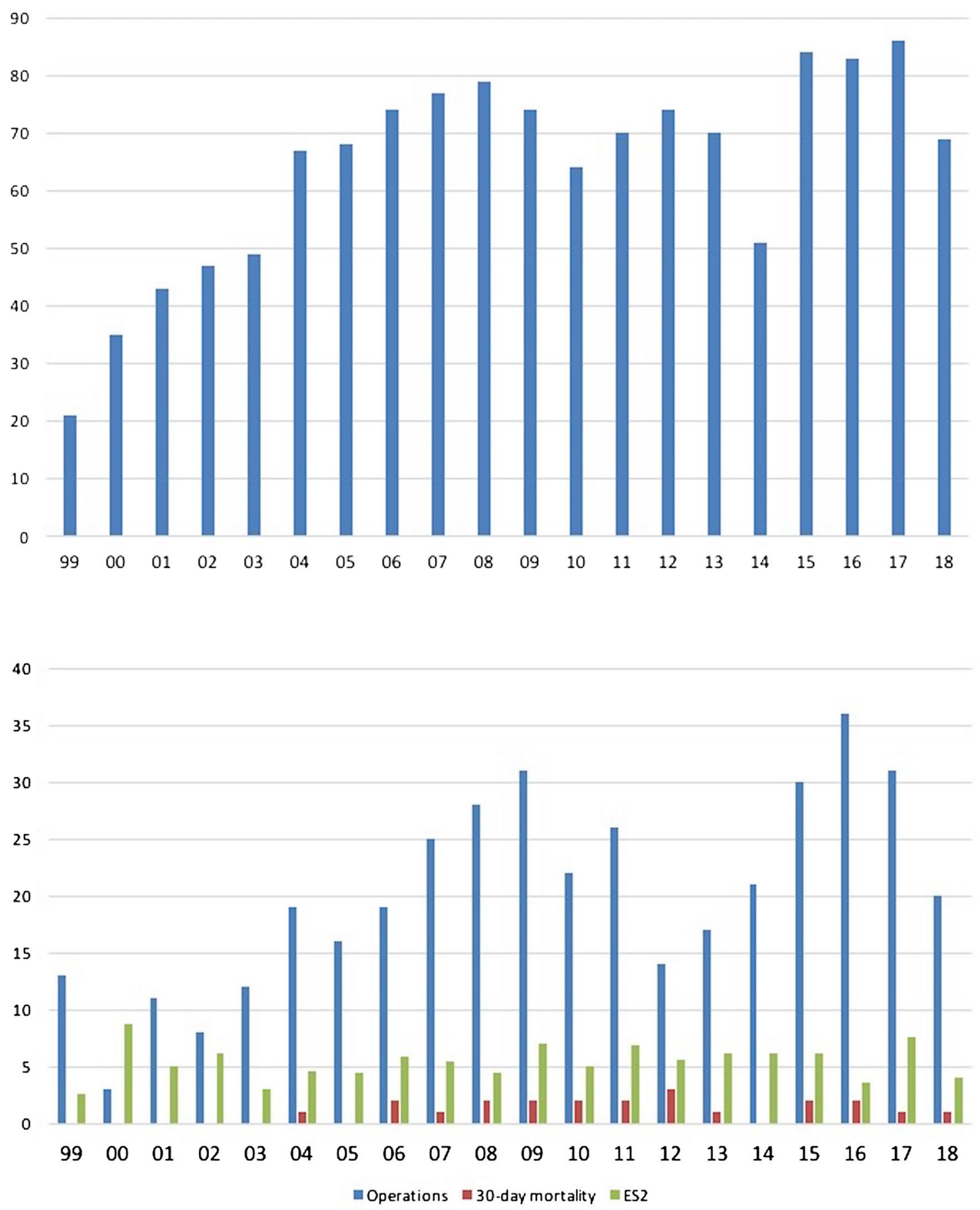

Fig. 1 Upper panel: year-by-year trend of all patients who underwent isolated aortic valve replacement or combined with other cardiac procedure. Lower panel: year-by-year trend of all patients included in the study population 


\section{Surgical technique}

All patients received standard anaesthesia. Median sternotomy was performed and cardiopulmonary bypass (CPB) established through ascending aorta and right atrial cannulation. Heparin was administered to achieve an activated clotting time $>480 \mathrm{~s}$ during $\mathrm{CPB}$. The heart was arrested with intermittent antegrade and retrograde cold blood cardioplegia. In patients undergoing AVR + CABG, circumflex marginal branches and right coronary artery distal coronary anastomoses were performed prior to AVR. The left internal thoracic artery was grafted to the left anterior descending artery following AVR. Proximal anastomoses were performed following aortotomy closure. We approached the aortic valve most commonly through a transverse aortotomy.

Aortic valve prosthesis selection was based on patient age, and patient or surgeon preference. The prosthetic valves were placed in a supra or infra-annular position according to the size of the annulus and the type of prosthesis.

Postoperatively, warfarin therapy was administered regardless of the type of aortic valve prosthesis implanted, unless there were specific contraindications to the use of warfarin. This was administered with a therapeutic international normalized ratio (INR) target between 2.0 and 3.0. Those patients who underwent the implantation of a bioprosthesis were switched over from warfarin therapy to $100 \mathrm{mg}$ of aspirin therapy at 3 months postoperatively, unless warfarin therapy was indicated postoperatively for persistent postoperative atrial fibrillation.

\section{Statistical analysis}

Categorical data were expressed as percentages; continuous data were expressed as mean \pm SD. Differences between $\mathrm{iAVR}$ and $\mathrm{AVR}+\mathrm{CABG}$ groups were compared with $X^{2}$ statistical test for categorical variables and $T$ test for continuous variables. To measure the treatment effect, odds ratios for binary and differences of means for continuous end points were calculated. Univariate and stepwise multivariate logistic regression analysis were performed to identify independent predictors of operative mortality and MACCE. A significance level of $p<0.25$ was used to enter variables into multivariate backward stepwise logistic regression analysis. A bootstrap technique (1000 resampling and respective multivariate analysis) was used for internal validation, and area under the curve (AUC) of the receiver operative characteristic (ROC) curves of both logistic regression and bootstrap were analysed to validate the model. Results are reported as percentages and odd ratio (OR) with $95 \%$ confidence intervals.

Long-term outcome was defined by all-cause mortality. This is an objective, unbiased end point, avoiding inaccurate, unsystematic and biased data derived from clinical documentation or medical data [22]. Death information was obtained from the Regional Institutional Health Database System for all patients. Long-term follow-up was calculated for patients who survived surgery and were discharged, from the time of operation to death or last follow-up. Follow-up was closed on November 17, 2018. Overall survival was estimated by the Kaplan-Meier method and expressed as percentage. The difference between the survival curves was assessed using log-rank test. To identify independent predictors of late mortality, univariate and multivariate Cox proportional hazards models were constructed. Covariates included in the model were determined a priori and included all the preoperative variables. A significance level of $p<0.25$ was used to enter variables in the Cox regression analysis. Due to differences in underlying patient characteristics, propensity matching of iAVR with AVR + CABG patients was employed. A propensity score (PS) was calculated and the resulting PS for each patient represented the probability of being in the iAVR or in the AVR + CABG group. Pretreatment variables were compared using the standardized mean difference (SMD) and a high quality of the matching was considered when the covariate balance between the groups showed an SMD less than $10 \%$. To neutralize the adverse effect of coronary artery disease, this covariate was not entered in the multivariable logistic regression model. Survival was compared for propensity-matched patients using the stratified log-rank test. All $p$ values $<0.05$ were considered statistically significant. All statistical analysis was performed using SPSS v.25.0 (Statistical Package for Social Science, SPSS Inc. Chicago, IL, USA) and Stata/MP 15.1 (Stata, Corp College Station, TX, USA).

The performance of EuroSCORE II model was evaluated analysing the discrimination power and calibration. The discrimination indicates how well the model separates patients who will die or survive at 30 days after surgery. Discrimination was evaluated by constructing a receiver operative characteristic (ROC) curves and calculating the area under the curve (c-statistic) with $95 \%$ confidence intervals (CI).

Calibration was evaluated by generating a calibration plot by quintile of scores for operative mortality that compare the prediction with the observed probability. The Fisher exact test was used to compare the observed and predicted operative mortality. Calibration was tested with the Hosmer-Lemeshow goodness-of-fit test which compares observed to predicted values by decile of predicted probability. Furthermore, the validity of the model was tested by calculating the Brier score, whose value is 0 when the prediction is perfect.

\section{Results}

The study population included 402 subjects stratified by treatment and divided into two groups: iAVR, $n=200$, and AVR + CABG, $n=202$. Demographic data and 
Table 1 Preoperative variables

\begin{tabular}{|c|c|c|c|c|}
\hline Variable & All patients $(n=402)$ & iAVR $(n=200)$ & $\mathrm{AVR}+\mathrm{CABG}(n=202)$ & $p$ \\
\hline Age & $78.1 \pm 2.7$ & $77.6 \pm 2.5$ & $78.5 \pm 2.8$ & $<0.001$ \\
\hline Male $(\%)$ & $193(48)$ & $77(38.5)$ & $116(57.4)$ & $<0.001$ \\
\hline Age $\geq 80$ years $(\%)$ & 119 (29.6) & $52(26)$ & $67(33.2)$ & 0.11 \\
\hline Weight (kg) & $69.8 \pm 12.9$ & $69.5 \pm 13.1$ & $70.1 \pm 12.8$ & 0.64 \\
\hline Height $(\mathrm{cm})$ & $164.4 \pm 8.5$ & $163.3 \pm 8.8$ & $165.5 \pm 8$ & 0.009 \\
\hline $\operatorname{BSA}\left(\mathrm{m}^{2}\right)$ & $1.74 \pm 0.19$ & $1.73 \pm 0.19$ & $1.75 \pm 0.18$ & 0.18 \\
\hline BMI & $25.7 \pm 4$ & $26 \pm 4$ & $25.5 \pm 3.9$ & 0.24 \\
\hline $\mathrm{BMI} \geq 30(\%)$ & $56(13.9)$ & $30(15)$ & $26(12.9)$ & 0.53 \\
\hline Hypertension (\%) & $328(81.6)$ & $162(81)$ & $166(82.2)$ & 0.76 \\
\hline Dyslipidaemia (\%) & $189(47)$ & $79(39.5)$ & $110(54.4)$ & 0.003 \\
\hline Diabetes $(\%)$ & $94(23.4)$ & $45(22.5)$ & $49(24.3)$ & 0.67 \\
\hline Diabetes on insulin (\%) & $29(7.2)$ & $10(5)$ & $19(9.4)$ & 0.08 \\
\hline Glucose & $106 \pm 34$ & $101.3 \pm 29.1$ & $112.5 \pm 38.8$ & 0.001 \\
\hline Smoke & $98(24.4)$ & $42(21)$ & $56(27.7)$ & 0.11 \\
\hline Atrial fibrillation & & & & 0.88 \\
\hline Paroxysmal (\%) & $36(9)$ & $17(8.5)$ & $19(9.4)$ & \\
\hline Persistent/permanent & $32(8)$ & $17(8.5)$ & $15(7.4)$ & \\
\hline Creatinine & $1.08 \pm 0.58$ & $1.03 \pm 0.48$ & $1.12 \pm 0.67$ & 0.14 \\
\hline Creatinine $\geq 2 \mathrm{mg} / \mathrm{dl}(\%)$ & $15(3.7)$ & $8(4)$ & $7(3.5)$ & 0.77 \\
\hline Creatinine clearance & $55.8 \pm 18.6$ & $56.9 \pm 19.3$ & $54.7 \pm 17.9$ & 0.24 \\
\hline Creatinine clearance severe $<50 \mathrm{ml} / \mathrm{min}(\%)$ & $155(38.6)$ & $70(45.2)$ & $85(54.8)$ & 0.14 \\
\hline Dialysis (\%) & $4(1 \%)$ & $2(1)$ & $2(1)$ & 0.91 \\
\hline Haemoglobin (g/dl) & $12.5 \pm 1.7$ & $12.4 \pm 1.7$ & $12.6 \pm 1.7$ & 0.33 \\
\hline Peripheral arteriopathy (\%) & $113(28.1)$ & $39(19.5)$ & $74(36.6)$ & $<0.001$ \\
\hline Poor mobility $(\%)$ & $14(3.5)$ & $7(3.5)$ & $7(3.5)$ & 0.98 \\
\hline CVE $(\%)$ & $32(8)$ & $14(7)$ & $18(8.9)$ & 0.47 \\
\hline Previous cardiac operation (\%) & $12(3)$ & $9(4.5)$ & $3(1.5)$ & 0.07 \\
\hline Previous CABG $(\%)$ & $7(1.7)$ & $6(3)$ & $1(0.5)$ & 0.05 \\
\hline Previous iAVR (\%) & $3(0.7)$ & $2(1)$ & $1(0.5)$ & 0.81 \\
\hline Previous AVR + CABG (\%) & $1(0.2)$ & $0(0)$ & $1(0.5)$ & 0.75 \\
\hline Previous other cardiac operation (\%) & $2(0.5)$ & $1(0.5)$ & $1(0.5)$ & 0.99 \\
\hline $\operatorname{COPD}(\%)$ & $54(13.4)$ & $28(14)$ & $26(12.9)$ & 0.74 \\
\hline Active endocarditis (\%) & $10(2.5)$ & $4(2)$ & $6(3)$ & 0.53 \\
\hline NYHA class & $2.14 \pm 0.77$ & $2.03 \pm 0.71$ & $2.25 \pm 0.81$ & 0.004 \\
\hline NYHA class 3-4 (\%) & $106(26.4)$ & $42(21)$ & $64(31.7)$ & 0.01 \\
\hline $\operatorname{CCS} 4(\%)$ & $29(7.2)$ & $4(2)$ & $25(6.2)$ & $<0.001$ \\
\hline Ejection fraction, $\%$ & $55.4 \pm 10.6$ & $56.7 \pm 9.3$ & $54 \pm 11.6$ & 0.01 \\
\hline Ejection fraction $\leq 50 \%(\%)$ & $98(24.4)$ & $38(38.8)$ & $60(61.2)$ & 0.01 \\
\hline Recent myocardial infarction (\%) & $19(4.7)$ & $2(1)$ & $17(8.4)$ & $<0.001$ \\
\hline PAPs $\geq 50 \mathrm{mmHg}(\%)$ & $42(10.4)$ & $24(12)$ & $18(8.9)$ & 0.31 \\
\hline Urgency operation (\%) & $38(9.5)$ & $6(3)$ & $32(15.8)$ & $<0.001$ \\
\hline Critical preoperative state (\%) & $6(1.5)$ & $1(0.5)$ & $5(2.5)$ & 0.10 \\
\hline EuroSCORE 2 & $5.4 \pm 5.8$ & $3.6 \pm 3.7$ & $7.2 \pm 6.9$ & $<0.001$ \\
\hline EuroSCORE $2>15 \%(\%)$ & $24(6)$ & $5(2.5)$ & $19(9.4)$ & 0.003 \\
\hline Aortic bicuspid valve (\%) & $7(1.7)$ & $5(2.5)$ & $2(1)$ & 0.24 \\
\hline Aortic valve disease & & & & 0.008 \\
\hline Stenosis (\%) & $301(74.9)$ & $150(75)$ & $151(74.8)$ & \\
\hline Insufficiency (\%) & $22(5.5)$ & $16(8)$ & $6(3)$ & \\
\hline Both stenosis and insufficiency (\%) & $79(19.7)$ & $34(17)$ & $45(22.3)$ & \\
\hline
\end{tabular}


Table 1 (continued)

\begin{tabular}{|c|c|c|c|c|}
\hline Variable & All patients $(n=402)$ & $\operatorname{iAVR}(n=200)$ & $\mathrm{AVR}+\mathrm{CABG}(n=202)$ & $p$ \\
\hline Aetiology of valve disease & & & & 0.05 \\
\hline Degenerative (\%) & $321(79.8)$ & $160(80)$ & $161(80.5)$ & \\
\hline Rheumatic (\%) & $51(12.7)$ & $20(10)$ & $21(10.4)$ & \\
\hline Endocarditis, active or chronic (\%) & $22(5.5)$ & $16(8)$ & $6(3)$ & \\
\hline Other $(\%)$ & $8(2)$ & $5(2.5)$ & $3(1.5)$ & \\
\hline No. of vessel disease & & & $1.9 \pm 0.8$ & \\
\hline Left main disease $(\%)$ & & & $32(15.8)$ & \\
\hline 1 vessel $(\%)$ & & & $70(34.7)$ & \\
\hline 2 vessels $(\%)$ & & & $70(34.7)$ & \\
\hline 3 vessels $(\%)$ & & & $62(30.7)$ & \\
\hline
\end{tabular}

$i A V R$ isolated aortic valve replacement, $C A B G$ coronary artery bypass grafting, $B S A$ body surface area, $B M I$ body mass index, $C V E$ cerebrovascular events, NYHA New York Heart Association, $C O P D$ chronic obstructive pulmonary disease (under long-term use of bronchodilators or steroids), CCS Canadian Cardiovascular Society, PAP pulmonary systolic arterial pressure

Table 2 Preoperative echocardiogram data

\begin{tabular}{lcccl}
\hline Variable & All patients $(n=402)$ & iAVR $(n=200)$ & $\begin{array}{l}\text { AVR +CABG } \\
(n=202)\end{array}$ & $p$ \\
\hline EF $(\%)$ & $55.4 \pm 10.6$ & $56.7 \pm 9.3$ & $54.1 \pm 11.6$ & 0.01 \\
Aortic peak gradient $(\mathrm{mmHg})$ & $77.6 \pm 25.5$ & $81.8 \pm 25.9$ & $73.3 \pm 24.5$ & 0.002 \\
Aortic mean gradient $(\mathrm{mmHg})$ & $48.5 \pm 17$ & $51.4 \pm 16.9$ & $45.4 \pm 16.7$ & 0.002 \\
Aortic valve area $\left(\mathrm{cm}^{2}\right)$ & $0.8 \pm 0.27$ & $0.78 \pm 0.26$ & $0.81 \pm 0.29$ & 0.39 \\
Aortic valve area index $\left(\mathrm{cm}^{2} / \mathrm{m}^{2}\right)$ & $0.46 \pm 0.16$ & $0.46 \pm 0.15$ & $0.47 \pm 0.16$ & 0.94 \\
Systolic septum wall thickness $(\mathrm{cm})$ & $1.44 \pm 0.31$ & $1.46 \pm 0.32$ & $1.41 \pm 0.28$ & 0.19 \\
Aortic valve regurgitation $($ grade $)$ & $1.11 \pm 1$ & $1.12 \pm 1.11$ & $1.15 \pm 0.98$ & 0.80 \\
PAP (mmHg) & $37.8 \pm 11.7$ & $38.7 \pm 12.4$ & $36.7 \pm 10.8$ & 0.16 \\
Mitral valve regurgitation (grade) & $1.2 \pm 0.6$ & $1.1 \pm 0.6$ & $1.2 \pm 0.6$ & 0.27 \\
\hline
\end{tabular}

$i A V R$ isolated aortic valve replacement, $C A B G$ coronary artery bypass grafting, $E F$ ejection fraction, $P A P s$ pulmonary systolic arterial pressure preoperative variables are outlined in Table 1 . The overall mean age was $78 \pm 2.7$ years, while AVR + CABG patients were older than iAVR patients (78.5 vs 77.6 years, respectively; $p<0.0001$ ). Patients undergoing combined AVR and $\mathrm{CABG}$ were also more likely males, with more baseline comorbidities such as elevated blood glucose level, peripheral artery disease, advanced New York Heart Association class and Canadian Cardiovascular Society class 4 , more recent myocardial infarction, more urgent operation and higher EuroSCORE II. The iAVR group had higher ejection fractions ( $56.7 \%$ vs $54 \%$, respectively; $p=0.028)$, greater aortic peak gradients $(81.8 \mathrm{mmHg}$ vs $73.3 \mathrm{mmHg}$, respectively; $p=0.002$ ), and greater aortic mean gradients $(51.4 \mathrm{mmHg}$ vs $45.4 \mathrm{mmHg}$, respectively; $p=0.002$ ). No differences were observed in aortic valve area, grade of myocardial hypertrophy, pulmonary artery pressure and mitral valve regurgitation grade (Table 2). In our institution, the TAVI program started in 2009. To verify whether any differences were reported among the patients during the study period, the study population was divided into two groups: pre-TAVI era (1999-2008;
Table 3 Outcomes according two different eras

\begin{tabular}{llll}
\hline Variables & $\begin{array}{l}\text { Era } \\
1999-2008 \\
(n=154)\end{array}$ & $\begin{array}{l}\text { Era } \\
2009-2018 \\
(n=248)\end{array}$ & $p$ \\
\hline Age & $78.3 \pm 2.8$ & $77.9 \pm 2.6$ & 0.09 \\
Over 80 years, $n(\%)$ & $49(31.8)$ & $70(28.2)$ & 0.44 \\
Male, $n(\%)$ & $66(42.9)$ & $127(51.2)$ & 0.10 \\
EuroSCORE II & $4.76 \pm 4.97$ & $5.86 \pm 6.33$ & 0.05 \\
EuroSCORE II $\geq 15 \%, n(\%)$ & $4(2.6)$ & $20(8.1)$ & 0.02 \\
30-day mortality, $n(\%)$ & $6(3.9)$ & $16(6.5)$ & 0.27 \\
MACCE, $n(\%)$ & $15(9.7)$ & $26(10.5)$ & 0.81 \\
iAVR & $77(50)$ & $123(49.6)$ & 0.93 \\
\hline
\end{tabular}

$i A V R$ isolated aortic valve replacement, MACCE major adverse cerebral cardiovascular events

$n=154)$ and post-TAVI era (2009-2018; $n=248)$. Table 3 reports some outcome and characteristics between the two eras. Among the variables analysed, we have observed a significant higher number of patients with EuroSCORE II $\geq 15 \%$ in patients of the $2009-2018$ era $(p=0.02)$, while 
the incidence of 30-day mortality and MACCE were comparable between the two eras.

\section{Perioperative data and early outcome}

The operative data are outlined in Table 4. As expected, aortic cross clamping (X-Clamp) time and cardiopulmonary bypass (CPB) time were significantly longer in AVR + CABG compared to iAVR subjects $(p<0.0001)$. Bioprosthetic valves were implanted in over $92 \%$ of patients in both groups. The most common valve size was $23 \mathrm{~mm}$ (39\% in iAVR group and $41.6 \%$ in AVR + CABG group). No difference was reported in the mean prosthesis size and mean prosthesis effective orifice area (EOA) between groups. Moderate patient-prosthesis mismatch (PPM), defined as EOA index $>0.85$ or $<0.65 \mathrm{~cm}^{2} / \mathrm{m}^{2}$, was reported in $8.7 \%$ of all subjects, without any difference between groups. The AVR + CABG group underwent a mean of $1.9 \pm 0.8$ distal anastomoses and 171 of 202 patients $(84.6 \%)$ had complete myocardial revascularization. $157(77.7 \%)$ underwent left internal thoracic artery to left anterior descending coronary artery anastomosis.

Operative mortality occurred in nine patients $(4.5 \%)$ in the iAVR group and in 13 patients $(6.5 \%)$ in the AVR + CABG group. The difference was not statistically significant $(p=0.38)$. Overall operative mortality was $5.5 \%$. Compared to the iAVR group, patients who underwent AVR + CABG needed more prolonged ventilator support ( $16.8 \%$ vs $7.5 \% ; p=0.004)$, required intraaortic balloon pump support ( $4 \%$ vs $0 \% ; p=0.004$ ), and suffered from low cardiac output syndrome $(11.4 \%$ vs $4.5 \% ; p=0.011)$ and new myocardial infarction $(3.5 \%$ vs $0.5 ; p=0.03)$. Moreover, MACCE was present more in the AVR + CABG group ( $16.8 \%$ vs $8 \%$, respectively; $p=0.007)$. Table 5 shows the early outcomes.

Table 4 Perioperative variables

\begin{tabular}{|c|c|c|c|c|}
\hline Variables & All patients $(n=402)$ & $\operatorname{AVR}(n=200)$ & $\mathrm{AVR}+\mathrm{CABG}(n=202)$ & $p$ \\
\hline Cardiopulmonary time (min) & $125.3 \pm 39.4$ & $107.6 \pm 26.8$ & $143.4 \pm 41.9$ & $<0.001$ \\
\hline Aortic cross-clamp time (min) & $94.7 \pm 29.8$ & $79.7 \pm 18.8$ & $110 \pm 31.3$ & $<0.001$ \\
\hline Number of distal anastomoses & & & $1.9 \pm 0.8$ & \\
\hline Type of prosthesis & & & & 0.32 \\
\hline Mechanical (\%) & $21(5.2)$ & $13(6.5)$ & $8(4)$ & \\
\hline Biological stented $(\%)$ & $381(94.8)$ & $187(93.5)$ & $194(96)$ & \\
\hline Mean prosthesis EOA $\left(\mathrm{cm}^{2} / \mathrm{m}^{2}\right)$ & $1.76 \pm 0.27$ & $1.76 \pm 0.27$ & $1.76 \pm 0.26$ & 0.94 \\
\hline $\mathrm{iEOA}\left(\mathrm{cm}^{2} / \mathrm{m}^{2}\right)$ & $1.01 \pm 0.15$ & $1.02 \pm 0.15$ & $1.01 \pm 0.14$ & 0.50 \\
\hline PPM grade & & & & 0.60 \\
\hline Normal $\left(P P M>0.85 \mathrm{~cm}^{2} / \mathrm{m}^{2}\right)(\%)$ & $366(91.3)$ & $184(92)$ & $182(90.5)$ & \\
\hline Moderate (PPM $\left.0.65-0.85 \mathrm{~cm}^{2} / \mathrm{m}^{2}\right)(\%)$ & $35(8.7)$ & $16(8)$ & $19(9.5)$ & \\
\hline Mean prosthesis size & $22.4 \pm 1.8$ & $22.3 \pm 1.8$ & $22.6 \pm 1.8$ & 0.17 \\
\hline Size $18(\%)$ & $1(0.2)$ & $1(0.5)$ & $0(0)$ & \\
\hline Size $19(\%)$ & $28(7)$ & $14(7)$ & $14(6.9)$ & \\
\hline Size $21(\%)$ & $137(34.6)$ & $74(37)$ & $63(31.2)$ & \\
\hline Size $23(\%)$ & $162(40.3)$ & $78(39)$ & $84(41.6)$ & \\
\hline Size $25(\%)$ & $59(14.7)$ & $27(13.5)$ & $32(15.8)$ & \\
\hline Size 27 (\%) & $15(3.7)$ & $6(3)$ & $9(4.5)$ & \\
\hline LAD territory target (\%) & & & $156(77.2)$ & \\
\hline Diagonal branch (\%) & & & $20(10)$ & \\
\hline Circumflex territory target (\%) & & & $109(54)$ & \\
\hline RCA territory target (\%) & & & $88(43.6)$ & \\
\hline LITA use (\%) & & & $157(77.7)$ & \\
\hline LITA on LAD (\%) & & & $156(77.2)$ & \\
\hline BITA $(\%)$ & & & $7(3.5)$ & \\
\hline RA use (\%) & & & $4(2)$ & \\
\hline $\operatorname{CMR}(\%)$ & & & $171(84.7)$ & \\
\hline
\end{tabular}

$i A V R$ isolated aortic valve replacement, $C A B G$ coronary artery bypass grafting, $i E O A$ indexed effective orifice area, $P P M$ prosthesis-patient mismatch, $L A D$ left anterior descending, $R C A$ right coronary artery, LITA left internal thoracic artery, BITA bilateral internal thoracic arteries, $R A$ radial artery, $C M R$ complete myocardial revascularization 
Table 5 Postoperative variables

\begin{tabular}{lllll}
\hline Variables & All patients $(n=402)$ & iAVR $(n=200)$ & $\begin{array}{l}\text { AVR }+ \text { CABG } \\
(n=202)\end{array}$ & $p$ \\
\hline Ventilator support $\geq 48 \mathrm{~h}(\%)$ & $49(12.2)$ & $15(7.5)$ & $34(16.8)$ & 0.004 \\
Tracheostomy (\%) & $4(1)$ & $1(0.5)$ & $3(0.7)$ & 0.32 \\
Intensive care unit stay (days) & $2.9 \pm 5.8$ & $2.7 \pm 6$ & $3.2 \pm 5.7$ & 0.41 \\
Re-exploration for bleeding (\%) & $24(6)$ & $14(7)$ & $10(5)$ & 0.38 \\
Neurologic injury & $13(3.2)$ & $4(2)$ & $9(4.5)$ & 0.16 \\
Acute renal failure (\%) & $30(7.5)$ & $12(6)$ & $18(8.9)$ & 0.17 \\
Haemodialysis (\%) & $9(2.2)$ & $6(3)$ & $3(1.5)$ & 0.30 \\
LCO syndrome (\%) & $32(8)$ & $9(4.5)$ & $23(11.4)$ & 0.01 \\
Sepsis (\%) & $21(5.2)$ & $9(4.5)$ & $12(5.9)$ & 0.51 \\
Acute lung injury (\%) & $24(6)$ & $13(6.5)$ & $11(5.4)$ & 0.65 \\
Pneumonia (\%) & $16(4)$ & $5(2.5)$ & $11(5.4)$ & 0.13 \\
Multi-organ failure (\%) & $8(2)$ & $3(1.5)$ & $5(2.5)$ & 0.48 \\
Myocardial infarction (\%) & $8(2)$ & $1(0.5)$ & $7(3.5)$ & 0.03 \\
Endocarditis & $3(0.7)$ & $1(0.5)$ & $2(1)$ & 0.56 \\
Atrial fibrillation (\%) & $183(45.5)$ & $95(47.5)$ & $88(43.6)$ & 0.42 \\
Required permanent PM (\%) & $12(3)$ & $8(4)$ & $4(2)$ & 0.23 \\
IABP (\%) & $8(2)$ & $0(0)$ & $8(4)$ & 0.004 \\
MACCE (\%) & $50(12.4)$ & $16(8)$ & $34(16.8)$ & 0.007 \\
Hospital stay & $10 \pm 8.9$ & $9.4 \pm 8.7$ & $10.7 \pm 8.7$ & 0.15 \\
30-day mortality (\%) & $22(5.5)$ & $9(4.5)$ & $13(6.5)$ & 0.38 \\
\hline & & & & \\
\hline
\end{tabular}

$i A V R$ isolated aortic valve replacement, $C A B G$ coronary artery bypass grafting, $L C O$ low cardiac output, $P M$ pace-maker, $I A B P$ intra-aortic balloon pump, $M A C C E$ major adverse cerebral cardiovascular events

Table 6 Multivariate analysis for 30-day mortality and in-hospital MACCE

\begin{tabular}{lrcc}
\hline Variables & Odd ratio & 95\% CI & $p$ value \\
\hline Multivariate for 30-day mortality & & \\
EuroSCORE II & 1.13 & $1.01-1.26$ & 0.01 \\
Postoperative stroke & 12.52 & $1.19-131.13$ & 0.03 \\
Low cardiac output & 8.72 & $2.45-30.99$ & 0.001 \\
Mechanical ventilation $>48 \mathrm{~h}$ & 8.92 & $2.65-30.01$ & $<0.001$ \\
Treatment (AVR + CABG) & 1.05 & $0.2-4.67$ & 0.94 \\
Multivariate for in-hospital MACCE & & \\
Preoperative CVE & 3.43 & $1.13-10.53$ & 0.03 \\
Creatinine (mg/dl) & 7.27 & $2.46-21.50$ & $<0.001$ \\
Cardiopulmonary bypass time & 1.01 & $1.004-1.033$ & 0.01 \\
Treatment (AVR+CABG) & 1.00 & $0.37-2.66$ & 0.98 \\
\hline
\end{tabular}

$C V E$ cerebrovascular events, $A V R$ aortic valve replacement, $C A B G$ coronary artery bypass grafting, $M A C C E$ major adverse cerebral cardiovascular event

Multivariate analysis identified EuroSCORE II [odd ratio (OR) 1.13 ; $95 \%$ confidence intervals $(\mathrm{CI}) 1.01-1.26$; $p=0.015]$, postoperative stroke (OR $12.53 ; 95 \%$ CI $1.19-131.13 ; p=0.035)$, postoperative low cardiac output syndrome (OR 8.72; 95\% CI 2.45-30.99; $p=0.001)$ and postoperative mechanical ventilation $>48 \mathrm{~h}(\mathrm{OR} 8.92 ; 95 \%$ CI $2.65-30.01 ; p<0.0001)$ as independent predictors of 30-day mortality. Treatment was not an independent predictor of 30-day mortality (Table 6). The areas under the curves were 0.88 (95\% CI 0.81-0.91; $p=0.002)$ and 0.89 (95\% CI $0.82-0.92 ; p=0.002$ ) for the logistic regression model and the bootstrap validation model, respectively.

Multivariate analysis identified preoperative cerebrovascular events (OR 3.43; 95\% CI 1.13-10.53; $p=0.029$ ), creatinine (OR 7.27; 95\% CI 2.46-21.50; $p<0.0001$ ) and CPB time (OR 1.01; 95\% CI 1.004-1.33; $p=0.011$ ) as independent predictors of in-hospital MACCE. Again, treatment was not an independent predictor of in-hospital MACCE (Table 6).

The area under the curve showed that the EuroSCORE model had a moderate discrimination power to predict the 30-day mortality with a $c$-statistic value of 0.73 (95\% CI 0.631-0.831) (Fig. 2a). Calibration plot by quintile of EuroSCORE II for 30-day mortality showed that for each quintile risk profile, the EuroSCORE II performed well (Fig. 2b). Discrimination and calibration analysis are shown in Table 7.

PS matching generated 138 matched pairs of patients. Figure 3 shows the balancing covariates before and after propensity score matching. Between the iAVR and AVR + CABG group, postoperative outcome in terms of re-exploration for bleeding ( $8 \%$ vs $4.3 \% ; 0.21)$, neurological adverse events $(1.4 \%$ vs $1.4 \% ; p>0.99)$, acute renal 
Fig. 2 a Calibration of 30-day mortality observed and predicted quintiles of EursoSCORE II. b Area under the curve for the EuroSCORE II
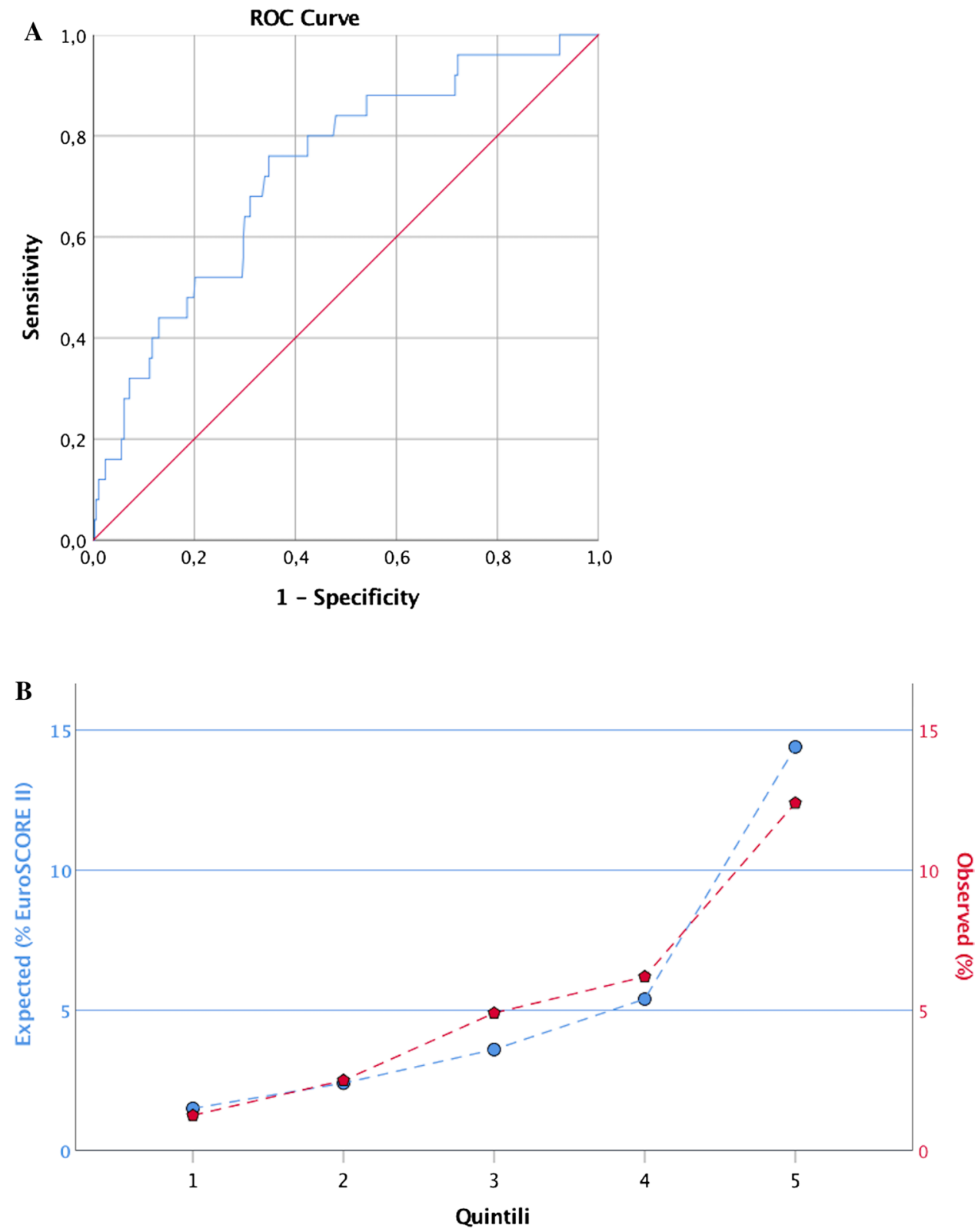

Table 7 Discrimination and calibration analysis for 30-day mortality

\section{Outcomes}

Predicted 30-day mortality (EuroSCORE II)

Discrimination for 30-day mortality

C-statistic (95\%, CI)

Calibration for 30-day mortality (4\%)

Observed/predicted ratio

Brier score

$5.4 \pm 5.8$

$0.73(0.631-0.831)$

Fisher's exact test $p$ value

0.74

0.05

0.83

Hosmer-Lemeshow test $p$ value (Chi square) failure $(4.3 \%$ vs $5.1 \% ; p=0.77)$, intensive care unit stay $(2.1 \pm 4.8$ days vs $2.7 \pm 5.2$ days; $p=0.33)$, hospital stay $(8.9 \pm 6.9$ days vs $9.6 \pm 7.3$ days; $p=0.33)$, atrial fibrillation $(47.1 \%$ vs $41.3 \% ; p=0.33)$ and 30 -day mortality $(2.9 \%$ vs $5.1 \% ; p=0.35)$ were comparable. IABP support ( $0 \%$ vs $2.9 \% ; p=0.04)$ and ventilator support $\geq 48 \mathrm{~h}(5.1 \%$ vs $11.6 \%$; $p=0.05)$ were more represented in iAVR patients. The early outcome between matched and unmatched populations was similar.

\section{Long-term results}

Three-hundred and eighty (94.5\%) patients survived hospital discharge beyond 30 days. Follow-up was achieved in 
Fig. 3 Love plot showing balancing covariates before and after propensity score matching. CCS Canadian Cardiovascular Society, NYHA New York Heart Association, $A M I$ acute myocardial infarction, $A F$ atrial fibrillation, $P A P s$ pulmonary artery systolic pressure, $B M I$ body mass index

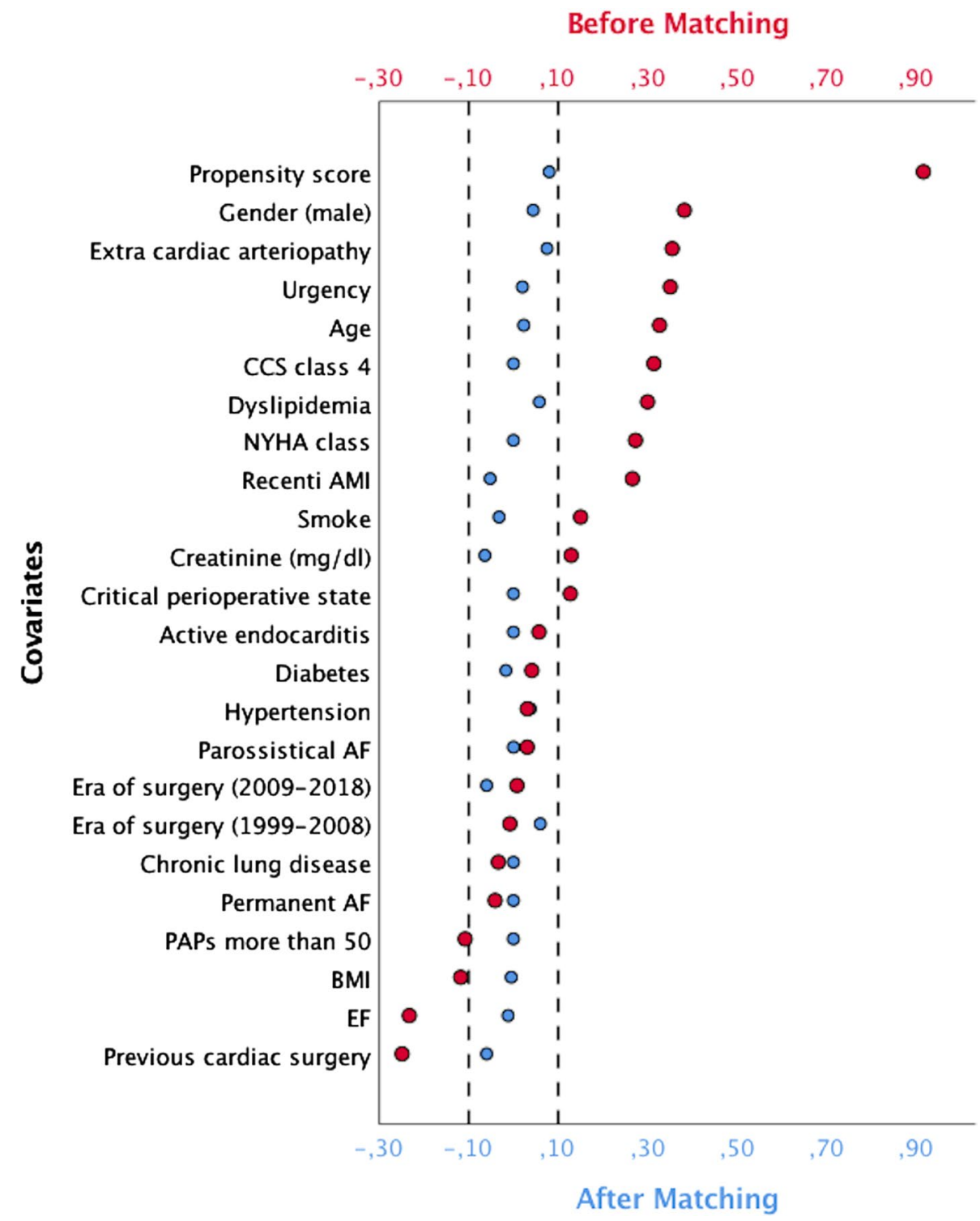

$100 \%$ of patients with a median follow-up of 8.08 (95\% CI 7.42-8.81) years, to a maximum of 16.1 years. Total followup had 6472 patient-years: 2914 patient-years for iAVR and 3252 patient-years in the AVR + CABG group.

One-hundred and eighty-six patients died at study completion $(48.9 \%$ ), with a mean survival of $7.9 \pm 0.3$ years, and median survival time of 8.08 (95\% CI 7.42-8.81) years. The median follow-up time for iAVR was 8.20 (95\% CI 7.56-8.81) years, with 108 patients (54\%) still alive. The median follow-up time for AVR + CABG was 7.91 (95\% CI 6.73-9.39) years with 108 patients (53.4\%) still alive.

Survival at 1,5 and 10 years was $94.7 \pm 1.6 \%$, $72.6 \pm 3.6 \%$ and $31.7 \pm 4.8 \%$ for patients included in the iAVR group and $89.1 \pm 2.3 \%, 73.9 \pm 3.5 \%$ and $37.2 \pm 4.8 \%$ for patients who underwent AVR combined with CABG. Long-term survival curves are shown in Fig. 4a. Log-rank test did not reveal any significant difference between groups $(p=0.99)$. Moreover, matched survival was similar between the two groups and the trend of matched survival curves was comparable with the unmatched population (Fig. 4b).

In Cox proportional hazard analysis, unadjusted concomitant AVR and CABG was not a predictor of late mortality [hazard ratio (HR) $0.99 ; 95 \%$ CI $0.74-1.33$; $p=0.99]$. Using adjusted multivariate analysis, creatinine (HR 1.50; 95\% CI 1.07-2.10; $p=0.018$ ), COPD (HR 1.97; 95\% CI 1.26-3.08; $p=0.003$ ) and NYHA class (HR 1.39; 95\% CI 1.530-7.816; $p<0.0001)$ were independent predictors of late mortality; the combined AVR + CABG was not associated with increased risk of late mortality (HR 0.83 ; 95\% CI 0.59-1.17; $p=0.30$ ) (Table 8). 

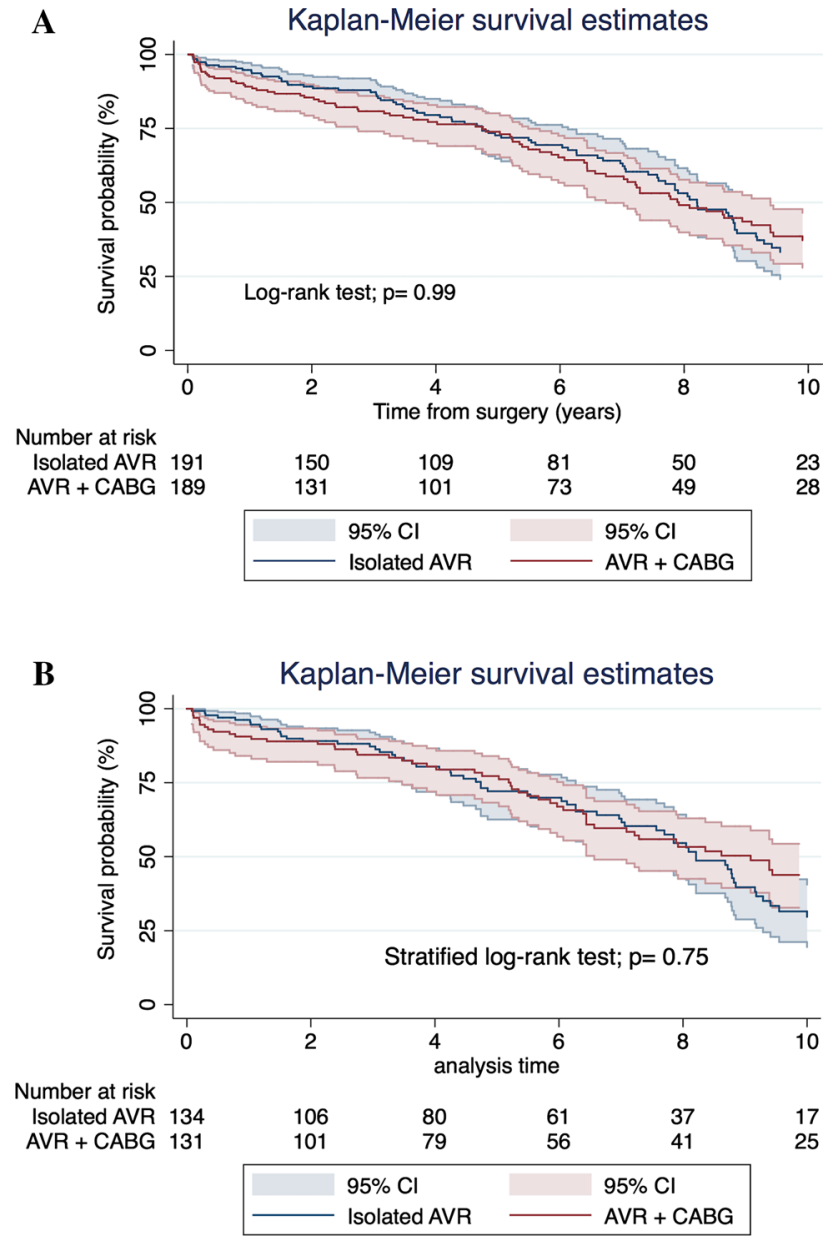

Fig. 4 a Kaplan-Meier event-free-survival analysis for all-cause of death for all patients who survived the operation. $A V R$ aortic valve replacement, $C A B G$ coronary artery bypass grafting. b Kaplan-Meier event-free-survival analysis for all-cause of death in matched patients who survived the operation. $A V R$ aortic valve replacement, $C A B G$ coronary artery bypass grafting

Table 8 Cox proportional hazard analysis for long-term mortality

\begin{tabular}{llll}
\hline Variables & Hazard ratio & $95 \%$ CI & $p$ value \\
\hline Unadjusted & & & \\
Treatment (AVR + CABG) & 0.99 & $0.74-1.33$ & 0.99 \\
Adjusted & & & \\
Creatinine (mg/dl) & 1.50 & $1.07-2.100$ & 0.01 \\
COPD & 1.97 & $1.26-3.08$ & 0.003 \\
NYHA class & 1.39 & $1.05-1.83$ & 0.01 \\
Treatment (AVR + CABG) & 0.83 & $0.59-1.17$ & 0.30 \\
\hline
\end{tabular}

$A V R$ aortic valve replacement, $C A B G$ coronary artery bypass grafting, $C O P D$ chronic obstructive pulmonary disease (under long-term use of bronchodilator or steroids), NYHA New York Heart Association

\section{Discussion}

Despite AVR + CABG patients being older than iAVR patients with greater comorbidity burden, and higher incidence of postoperative complications, no significant difference in early and long-term outcomes was identified between groups. Addition of CABG to AVR was not an independent risk factor for 30-day mortality, in-hospital MACCE, or long-term survival.

Increased life expectancy, combined with improved surgical techniques and postoperative care, has progressively expanded the criteria of cardiac surgery operability in the elderly. Almost half of the elderly patients with surgical aortic valve disease have associated coronary artery disease. The growth of minimally invasive techniques has made treatment choices increasingly challenging [5].

Multiple studies have confirmed that isolated AVR can be performed with good outcomes in terms of early and late mortality and morbidity in the elderly [5, 23, 24]. Furthermore, minimally invasive AVR can be achieved in elderly patients with satisfactory early and mid-term results $[25,26]$. Results after combined CABG + AVR in the elderly population remain unclear. Various reports have suggested iAVR in the elderly to be associated with lower risk than AVR combined with CABG [11, 27, 28]

Recent data from the German Aortic Valve Registry reported [14] encouraging in-hospital mortality of $2.3 \%$ in 5738 patients over 75 years who underwent isolated AVR compared to $3.6 \%$ for AVR + CABG in the same age group. They observed low overall complication rates and good 1-year survival for all risk groups of patients, corroborating that conventional surgical AVR, with or without CABG, remains the gold standard for patients at low and intermediate risk.

In this study, 30-day in-hospital mortality was not statistically different between iAVR and AVR + CABG (4.5\% and $6.5 \%$, respectively; $p=0.38$ ). These results are consistent with previous studies comparing both treatments that reported in-hospital mortality in iAVR ranging from 0 to $7.6 \%$ and AVR + CABG ranging from 1.9 to $11.5 \%$ [9, $13-15,17,19,21,29,30]$.

Our data show that CABG combined with AVR can be safely performed without increasing operative mortality. Multivariate logistic regression of 30-day mortality found preoperative comorbidity such as EuroSCORE II, and postoperative complications such as stroke, low cardiac output and mechanical ventilation more than $48 \mathrm{~h}$, as independent predictors of in-hospital mortality. The model revealed some predictors such as low cardiac output and mechanical ventilation more than $48 \mathrm{~h}$ which are wellknown risk factors for early death. 
CPB time and X-Clamp duration were statistically different between groups as expected, higher in AVR + CABG than iAVR subjects. These same differences are reported in other studies. Longer duration of CPB and X-Clamp are always a concern, particularly in the elderly, as longer CPB is associated with increased incidence of cerebral, renal and coagulation damage, and greater X-Clamp predisposes to increased risk of myocardial damage. Cappabianca [16] reported that the duration of CPB time (OR 1.02, $p=0.004$ ) was an independent predictor of in-hospital mortality, while Dell'Amore [19] observed that a shorter X-Clamp time had a protective role (OR $0.8, p=0.01$ ).

In this study, both CPB and X-Clamp times were not associated with increased 30-day surgical mortality. Superior biocompatible material technology for CPB and improved myocardial protection are advances that permit long and complex cardiac operations, even in very elderly patients. We advocate complete myocardial revascularization as paramount to achieving good results and optimizing perioperative care.

Our reported 30-day mortality for iAVR $(4.5 \%)$ and AVR + CABG (6.5\%) are lower than in-hospital mortality reported by other investigators during the last decade [10, $11,16,29]$.

The most frequent postoperative complication observed was new-onset atrial fibrillation (NOAF). The overall rate was $45.5 \%$, similar between the iAVR and AVR + CABG groups ( $47.5 \%$ vs $43.6 \%$, respectively; $p=0.42$ ). New-onset AF was not associated with 30-day mortality. Most cases of postoperative NOAF converted to sinus rhythm after medical treatment with amiodarone or with electrical cardioversion (6.4\%). Among patients with postoperative NOAF, only 31 (7.7\%) were discharged with permanent atrial fibrillation.

Long-term survival reported in our study is one of the longest follow-ups (up to 16 years) described in the literature for elderly patients ( $\geq 75$ years) who underwent iAVR or combined AVR $+\mathrm{CABG}$. Survival at 1,5 and 10 years was $94.7 \pm 1.6 \%, 72.6 \pm 3.6 \%$ and $31.7 \pm 4.8 \%$ for iAVR and $89.1 \pm 2.3 \%, 73.9 \pm 3.5 \%$ and $37.2 \pm 4.8 \%$ for AVR $+\mathrm{CABG}$ patients. There is no statistical difference in survival curves between the two groups. These survival rates are comparable with previous reports [9, 18-20], and good long-term survival is evident despite advanced age.

Cox regression multivariate analysis demonstrated that factors such as creatinine blood level, creatinine clearance $\leq 50 \mathrm{ml} / \mathrm{min}, \mathrm{COPD}$ and NYHA class were independent predictors of late mortality [31].

Noteworthy is the lack of statistical difference between iAVR and AVR + CABG with respect to long-term survival. This result is in line with other reports [9, 12, 19-21], but in contrast with the results described by Melby [10] and Molstad [32]. On one hand, Melby et al. observed that performing an AVR with concomitant CABG provided an improved long-term survival compared with isolated AVR (HR 0.7, 95\% CI 0.47-0.96; $p=0.020)$. On the other side, Molstad and colleagues reported a detrimental effect of concurrent CABG at the time of AVR surgery compared to patients who underwent iAVR on long-term survival (HR 1.37, 95\% CI $1.08-1.73 ; p=0.02$ ).

We found addition of CABG at the time of AVR was not associated with increased mortality in the unadjusted (HR $0.99 ; 95 \%$ CI $0.74-1.33 ; p=0.99$ ) and adjusted analysis (HR $0.83 ; 95 \%$ CI $0.59-1.17 ; p=0.30$ ). Surgical revascularization improves myocardial metabolism and reduces the risk of ischemia in hypertrophied left ventricles suggesting that CABG combined with AVR should be performed when indicated even in the elderly, without increasing operative risk.

In our study population, propensity matching provides an opportunity to compare the outcomes of patients with iAVR with those of patients with AVR + CABG and otherwise similar non-CAD comorbidity profiles. Matched and unmatched survival curves had similar trend, suggesting that CABG at the time of AVR neutralizes the adverse effects of coronary disease, provided that ischemic myocardial damage has not occurred after the operation. However, this data should be considered with caution because propensity score matching selected 276 subjects among 402 patients (68.6\%), meaning that the best and the worst patients might have been excluded from the PSM analysis.

TAVI has been dramatically increased during the last decade [7] and, recently, indication of TAVI has expanded to nonagenarians [33] and even to intermediate-risk patients [34]. In a recent meta-analysis, Biancari et al. [33] reported a 30-day mortality of $7.1 \%$ and an excellent mid-term survival of $61.3 \%$ at 3 years in a pool of 1227 nonagenarians who underwent TAVI, while Thourani et al. [34] reported a 30-day mortality of $1.1 \%$ and low incidence of mortality, stroke and aortic regurgitation at 1 year in a population of 1077 intermediate-risk patients. TAVI is now considered the first treatment of choice for high-risk and elderly patients. However, patients with TAVI indications need to be carefully selected because of some anatomical reasons, such as low take-off of coronary ostia, aggressive peripheral arteriopathy and left ventricular obstruction or calcification, which may severely compromise the immediate results. Moreover, the valve-in-valve option has considerable limitations in patients with small bioprosthetic valve and is not indicated in patients with a mechanical valve [35]. Compared with TAVI, the surgical approach has the advantage of removing the calcified native valve, making a careful debridement of the aortic annulus and sizing appropriately the prosthesis valve. These factors may contribute to reducing the risk of paravalvular leak, PPM and requirement of permanent pacemaker. A controversial topic concerns the short and midterm results of those patients who underwent TAVI + PCI compared to patients undergoing CABG + AVR. Despite 
that isolated TAVI proved firstly to have better results in those patients without previous CABG compared to patients with previous CABG [36] and secondly to be superior compared to patients who underwent TAVI + PCI [37], the evidence of a superiority of the TAVI + PCI compared to CABG + AVR is still very controversial and few studies have investigated it so far. Wendt et al. [38] reported comparable results in high-risk patients undergoing either TAVI + PCI or AVR + CABG showing a 30-day mortality rate of $11.9 \%$ vs $12.5 \%(p=0.89)$, respectively, and a 4-year survival of 53\% and by $60.7 \%(p=0.191)$, respectively. Similar results have recently been reported by Barbanti et al. [39] in a retrospective study of intermediate-risk population. A more recent prospective randomized study (SURTAVI trial) [40] showed acceptable and comparable results among intermediate-risk patients who underwent either TAVI + PCI or CABG + AVR in terms of all-cause mortality and disabling stroke at 2 years (16\%, vs $14 \%, p=0.69$, respectively).

Our results showed a moderate discrimination power and a good calibration for 30-day mortality of EuroSCORE II. Conflicting results have been reported for EuroSCORE II to overestimate [41] and underestimate the risks [42]. Therefore, we can only recognize that the accuracy of the model is validated in our study population and we must be extremely cautious in stating that our discrimination and calibration analysis is better or worse than others already reported in the literature.

\section{Study limitations}

This study is limited in its retrospective nature. Moreover, this is a single-centre study with a relatively small sample size analysed in a long period. We know that this limitation may be as cause of preoperative surgical selection bias due to the change over the study period of cardiologist's referral, the surgeon's decision to operate some elderly patients and the development of TAVI techniques.

Since the beginning of TAVI program in 2009 in our institution, patients older than 80 years with high operative risk (EuroSCORE II $>15 \%$ ), patients with high grade of frailty and patients who refused the conventional surgical approach were directed to the TAVI strategy. This has obviously changed the characteristics of our cohort study during the last decade, creating bias in patient selection.

Patients older than 75 years may be affected by different grades of frailty. Unfortunately, we could not account for this variable that may affect the early and long-term results and can be one more factor causing preoperative surgical selection bias.

Another limitation relates to the long-term follow-up, which is restricted only to the all-cause death.

\section{Conclusions}

This study represents one of the longest reported follow-up periods for iAVR and AVR + CABG in elderly patients. Although patients over 75 years of age underwent combined surgical myocardial revascularization and AVR had higher incidence of early complications, this treatment does not represent a prohibitive surgical risk in this population. Long-term outcomes are comparable to elderly patients who underwent isolated aortic valve replacement.

Acknowledgements The authors wish to thank Giorgia Pavan for her tireless and high value contribution during the correction and writing of the article.

Funding This work was supported by the University of Milano-Bicocca, Fondi Ateneo Ricerca, F.A.R. [Grant number 2011-ATE-0432].

\section{Compliance with ethical standards}

Conflict of interest All authors declare that they have no conflict of interest.

\section{References}

1. World Health Organization (2012) 10 facts on ageing and the life course. https://www.who.int/features/factfiles/ageing/agein g_facts/en/index.html. Accessed 2 Feb 2014

2. https://ec.europa.eu/eurostat/statistics-explained/image s/8/85/Population_structure_by_major_age_groups $\% 2 C$ CEU28\%2C_2016-80_\%28\%25_of_total_population\%29.png. Accessed 11 Jan 2019

3. Barreto-Filho JA, Wang Y, Dodson J, Desai MM, Sugeng L, Geirsson A, Krumholz HM (2013) Trends in aortic valve replacement for elderly patients in the United States, 19992011. JAMA 310:2078-2085

4. Vasques F, Lucenteforte E, Paone R, Mugelli A, Biancari F (2012) Outcome of patients aged $\geq 80$ years undergoing combined aortic valve replacement and coronary artery bypass grafting: a systematic review and meta-analysis of 40 studies. Am Heart J 164:410-418.e1

5. Ashikhmina EA, Schaff HV, Dearani JA, Sundt TM, Suri RM, Park SJ, Burkhart HM, Li Z, Daly RC (2011) Aortic valve replacement in the elderly determinants of late outcome. Circulation 124:1070-1078

6. Baumgartner H, Falk V, Bax JJ, De Bonis M, Hamm C, Holm PJ, Iung B, Lancellotti P, Lansac E, Munoz DR, Rosenhek R, Sjogren J, Mas PT, Vahanian A, Walther T, Wendler O, Windecker S, Zamorano JL (2017) 2017 ESC/EACTS Guidelines for the management of valvular heart disease. Eur Heart J 38:2739-2791

7. Garcia S, Kelly R, Mbai M, Gurevich S, Oestreich B, Yannopoulos D, Adabag S (2018) Outcomes of intermediate-risk patients treated with transcatheter and surgical aortic valve replacement in the Veterans Affairs Healthcare System: a single center 20-year experience. Catheter Cardiovasc Interv 92:390-398

8. Chakos A, Wilson-Smith A, Arora S, Nguyen TC, Dhoble A, Tarantini G, Thielmann M, Vavalle JP, Wendt D, Yan TD, Tian DH (2017) Long term outcomes of transcatheter aortic valve 
implantation (TAVI): a systematic review of 5-year survival and beyond. Ann Cardiothorac Surg 6:432-443

9. Fukui T, Bando K, Tanaka S, Uchimuro T, Tabata M, Takanashi S (2014) Early and mid-term outcomes of combined aortic valve replacement and coronary artery bypass grafting in elderly patients. Eur J Cardiothorac Surg 45:335-340

10. Melby SJ, Zierer A, Kaiser SP, Guthrie TJ, Keune JD, Schuessler RB, Pasque MK, Lawton JS, Moazami N, Moon MR, Damiano RJ $\mathrm{Jr}$ (2007) Aortic valve replacement in octogenarians: risk factors for early and late mortality. Ann Thorac Surg 83(5):1651-1656 (discussion 1656-1657)

11. Kolh P, Kerzmann A, Honore C, Comte L, Limet R (2007) Aortic valve surgery in octogenarians: predictive factors for operative and long-term results. Eur J Cardiothorac Surg 31:600-606

12. Matsuura K, Ueda H, Kohno H, Tamura Y, Watanabe M, Inui T, Inage Y, Yakita Y, Matsumiya G (2018) Does the presence of coronary artery disease affect the outcome of aortic valve replacement? Heart Vessels 33:1-8

13. Koyama T, Sawada K, Goto Y, Ogawa S, Baba H, Okawa Y (2015) Early and mid-term results of isolated aortic valve replacement for aortic stenosis in octogenarians. Gen Thorac Cardiovasc Surg 63:216-221

14. Holzhey D, Mohr FW, Walther T, Möllmann H, Beckmann A, Kötting J, Figulla HR, Cremer J, Kuck KH, Lange R, Sack S, Schuler G, Beyersdorf F, Böhm M, Heusch G, Meinertz T, Neumann T, Papoutsis K, Schneider S, Welz A, Hamm CW (2016) Current results of surgical aortic valve replacement: insights from the german aortic valve registry. Ann Thorac Surg 101:658-666

15. Abel NJ, Rogal GJ, Burns P, Saunders CR, Chamberlain RS (2013) Aortic valve replacement with and without coronary artery bypass graft surgery in octogenarians: is it safe and feasible? Cardiology 124:163-173

16. Cappabianca G, Ferrarese S, Musazzi A, Terrieri F, Corazzari C, Matteucci M, Beghi C (2016) Predictive factors of long-term survival in the octogenarian undergoing surgical aortic valve replacement: 12-year single-centre follow-up. Heart Vessels 31:1798-1805

17. Florath I, Albert A, Boening A, Ennker IC, Ennker J (2010) Aortic valve replacement in octogenarians: identification of high-risk patients. Eur J Cardiothorac Surg 37:1304-1310

18. Beach JM, Mihaljevic T, Svensson LG, Rajeswaran J, Marwick T, Griffin B, Johnston DR, Sabik JF III, Blackstone EH (2013) Coronary artery disease and outcomes of aortic valve replacement for severe aortic stenosis. J Am Coll Cardiol 61:837-848

19. Dell'Amore A, Aquino TM, Pagliaro M, Lamarra M, Zussa C (2012) Aortic valve replacement with and without combined coronary bypass grafts in very elderly patients: early and long-term results. Eur J Cardiothorac Surg 41:491-498

20. Sasaki Y, Hirai H, Hosono M, Bito Y, Nakahira A, Suehiro Y, Kaku D, Okada Y, Suehiro S (2013) Adding coronary artery bypass grafting to aortic valve replacement increases operative mortality for elderly (70 years and older) patients with aortic stenosis. Gen Thorac Cardiovasc Surg 61:626-631

21. Wang TK, Choi DH, Ramanathan T, Ruygrok PN (2017) Aortic valve replacement with or without concurrent coronary artery bypass grafting in octogenarians: 8-year cohort study. Heart Lung Circ 26:82-87

22. Lauer MS, Blackstone EH, Young JB, Topol EJ (1999) Cause of death in clinical research: time for a reassessment? J Am Coll Cardiol 34:618-620

23. Klomp WWJ, Nierich AP, Peelen LM, Bruinsma GJ, Dambrink JHE, Moons KGM, van't Hof AWJ (2016) Survival and quality of life after surgical aortic valve replacement in octogenarians. J Cardiothorac Surg 11:38

24. Di Eusanio M, Fortuna D, Cristell D, Pugliese P, Nicolini F, Pacini D, Gabbieri D, Lamarra M (2012) Contemporary outcomes of conventional aortic valve replacement in 638 octogenarians: insights from an Italian Regional Cardiac Surgery Registry (RERIC). Eur J Cardiothorac Surg 41:1247-1253

25. Totsugawa T, Hiraoka A, Tamura K, Yoshitaka H, Sakaguchi T (2019) Minimally invasive aortic valve replacement through a right anterolateral mini-thoracotomy for the treatment of octagenarians with aortic valve stenosis. Heart Vessels 34:462-469

26. Chang C, Raza S, Altarabsheh SE, Delozier S, Sharma UM, Zia A, Khan MS, Neudecker M, Markowitz AH, Sabik JF 3rd, Deo SV (2018) Minimally invasive approach to surgical aortic valve replacement: a meta-analysis. Ann Thorac Surg 106:1881-1889

27. Likosky DS, Sorensen MJ, Dacey LJ, Baribeau YR, Leavitt BJ, DiScipio AW, Hernandez F Jr, Cochran RP, Quinn R, Helm RE, Charlesworth DC, Clough RA, Malenka DJ, Sisto DA, Sardella G, Olmstead EM, Ross CS, O'Connor GT (2009) Long-term survival of the very elderly undergoing aortic valve surgery. Circulation 120:S127-133

28. Carnero-Alcázar M, Reguillo-Lacruz F, Alswies A, VillagránMedinilla E, Maroto-Castellanos LC, Rodríguez-Hernández J (2010) Short- and midterm results for aortic valve replacements in octagenarias. Interact Cardiovasc Thorac Surg 10:549-554

29. Harris RS, Yan TD, Black D, Bannon PG, Bayfield MS, Hendel PN, Wilson MK, Vallely MP (2013) Outcomes of surgical aortic valve replacement in octogenarians. Heart Lung Circ 22:618-626

30. Langanay T, Flécher E, Fouquet O, Ruggieri VG, De La Tour B, Lelong B, Verhoye JP, Corbineau H, Leguerrier A (2012) Aortic valve replacement in the elderly: the real life. Ann Thorac Surg 93:70-77

31. Ho E, Mathur MN, Brady PW, Marshman D, Brereton RJ, Ross DE, Bhindi R, Hansen PS (2014) Surgical aortic valve replacement in very elderly patients aged 80 years and over: evaluation of early clinical outcomes. Heart Lung Circ 23:242-248

32. Mølstad P, Veel T, Rynning S (2012) Long-term survival after aortic valve replacement in octogenarians and high-risk subgroups. Eur J Cardiothorac Surg 42:934-940

33. Biancari F, D'Errigo P, Rosato S, Pol M, Tamburino C, Ranucci M, Seccareccia F (2017) Transcatheter aortic valve replacement in nonagenarians: early and intermediate outcome from the observant study and meta-analysis of the literature. Heart Vessels 32:157-165

34. Thourani VH, Kodali S, Makkar RR, Herrmann HC, Williams M, Babaliaros V, Smalling R, Lim S, Malaisrie SC, Kapadia S, Szeto WY, Greason KL, Kereiakes D, Ailawadi G, Whisenant BK, Devireddy C, Leipsic J, Hahn RT, Pibarot P, Weissman NJ, Jaber WA, Cohen DJ, Suri R, Tuzcu EM, Svensson LG, Webb JG, Moses JRW, Mack MJ, Mille DG, Smith CR, Alu MC, Parvataneni R, D'Agostino RB Jr, Leon MB (2016) Transcatheter aortic valve replacement versus surgical valve replacement in intermediate-risk patients: a propensity score analysis. Lancet 387(10034):2218-2225

35. Eggebrecht H, Schäfer U, Treede H, Boekstegers P, Babin-Ebell J, Ferrari M, Möllmann H, Baumgartner H, Carrel T, Kahlert P, Lange P, Walther T, Erbel R, Mehta RH, Thielmann M (2011) Valve-in-valve transcatheter aortic valve implantation for degenerated bioprosthetic heart valves. JACC Cardiovasc Interv 4:1218-1227

36. Kawashima H, Watanabe Y, Kozuma K, Kataoka A, Nakashima M, Hioki H, Nagura F, Nara Y, Shirai S, Tada N, Araki M, Naganuma T, Yamanaka F, Ueno H, Tabata M, Mizutani K, Higashimori A, Takagi K, Yamamoto M, Hayashida K, OCEAN-TAVI Investigators (2018) Comparison of midterm outcomes of transcatheter aortic valve implantation in patients with and without previous coronary artery bypass grafting. Heart Vessels 33:1229-1237

37. Kotronias RA, Kwok CS, George S, Capodanno D, Ludman PF, Townend JN, Doshi SN, Khogali SS, Généreux P, Herrmann HC, 
Mamas MA, Bagur R (2017) Transcatheter aortic valve implantation woth or without percutaneous coronary artery revascularization strategy: a systematic review and meta-analysys. J Am Heart Assoc 6:e005960

38. Wendt D, Kahlert P, Lenze T, Neuhauser M, Price V, Konorza T, Erbel R, Jakob H, Thielmann M (2013) Management of high-risk patients with aortic stenosis and coronary artery disease. Ann Thorac Surg 95:599-605

39. Barbanti M, Buccheri S, Capodanno D, D'Errigo P, Ranucci M, Rosato S, Santoro G, Fusco D, Tamburino C, Biancari F, Seccareccia F, OBSERVANT Research Group (2018) Transcatheter or surgical treatment of severe aortic stenosis and coronary artery disease: a comparative analysys from the Italian OBSERVANT study. Int J Cardiol 270:102-106

40. Søndergaard L, Popma JJ, Reardon MJ, Van Mieghem NM, Deeb GM, Kodali S, George I, Williams MR, Yakubov SJ, Kappetein AP, Serruys PW, Grube E, Schiltgen MB, Chang Y, Engstrøm T, SURTAVI Trial Investigators (2019) Comparison of a complete percutaneous versus surgical approach to aortic valve replacement and revascularization in patients at intermediate surgical risk: results from the randomized SURTAVI trial. Circulation. https:// doi.org/10.1161/CIRCULATIONAHA.118.039564

41. Barili F, Pacini D, Capo A, Rasovic O, Grossi C, Alamanni F, Di Bartolomeo R, Parolari A (2013) Does EuroSCORE II perform better than its original versions? A multicentre validation study. Eur Heart J 34:22-29

42. Grant SW, Hickey GL, Dimarakis I, Trivedi U, Bryan A, Treasure T, Cooper G, Pagano D, Buchan I, Bridgewater B (2012) How does EuroSCORE II perform in UK cardiac surgery: an analysis of 23,740 patients from the Society for Cardiothoracic Surgery in Great Britain and Ireland National Database. Heart 98:1568-1572

Publisher's Note Springer Nature remains neutral with regard to jurisdictional claims in published maps and institutional affiliations. 